\title{
MACROEVOLUTIONARY PATTERNS IN BRYOZOANS
}

\author{
KEY*, Marcus M., Jr., Department of Geology, Dickinson College, P.O. Box 1773, Carlisle, PA \\ 17013-2896, U.S.A.
}

What controls the diversity of life? Is it the randomness of historical contingency or the deterministic constraints of adaptive design? Several recent studies of colonial animals have suggested that nonrandom macroevolutionary patterns are common in the fossil record. These patterns generally consist of long-term, interspecific improvements in the biomechanical design and/or ecological success of some lineages over others. The problem with these previous studies is that they simply censused morphology through time. That is, they read the stratigraphic record directly. What this study does is examine macroevolutionary morphologic patterns within a phylogenetically well-defined lineage of lower Paleozoic trepostome bryozoans.

17 species of ramose trepostomes ranging from the Middle Ordovician to the Upper Silurian were examined. 16 ingroup species were analyzed: 7 species of halloporids (including species belonging to Hallopora, Parvohallopora, and Tarphophragma) and 8 species of bimuroporids (including species belonging to Bimuropora and Champlainopora). Two species of Eridotrypa were chosen for the outgroup. These ingroup taxa were selected as they are united by several synapomorphs strongly suggesting this lineage is monophyletic. Phylogenetic hypotheses were constructed from a cladistic analysis of 40 multistate, qualitative, morphologic characters using outgroup analysis and the parsimony principle. This led to a single most parsimonious cladogram of 78 steps with a consistency index of 0.641 .

Within this lineage, species were examined for changes in the distribution of calcite across the colony by measuring a single, continuously varying, quantitative, morphologic character. Calcite distribution across cylindrical branching colonies has been shown theoretically by Wainwright et al. and empirically by Cheetham to affect branch strength. Calcite distribution was measured by exozone width. The exozone is the peripheral part of the colony where most of the calcite is precipitated due to the thick zooecial walls there. Exozone width is proportional to branch strength. This morphometric character is relatively independent of the qualitative characters used in the cladistic analysis.

Exozone width was measured using a personal computer-based, digital, image analysis system. To minimize operator and system error as well as to ensure that intracolony and intraspecific variation was sufficiently represented, exozone width was measured 10 times per colony. These values were then averaged to obtain colony means. The colony means were averaged to obtain species means. A total of 261 colonies belonging to the 17 species were measured.

Macroevolutionary patterns were quantified by comparing the mean exozone width for each species to 3 different evolutionary metrics derived from the most parsimonious cladogram: number of branching events each species has undergone from the base of the cladogram, number of character state transformations each species has undergone from the base of the cladogram, and the mean manhattan distance between each species and the 2 outgroup species.

Regardless of which measure of evolution is chosen, the results indicate that exozone width has significantly increased through this lineage. Linear regressions revealed that these 3 trends are significant at $\mathbf{P}=0.05$. That is, the evolutionary history of this lineage reveals a progressive, nonrandom increase in branch strength. Many more species within this lineage need to be analyzed. This pattern is significant with 17 species; it may or may not be when the project is completed with the estimated 100 species that will eventually be measured. It must also be remembered that the patterns observed are dependent on the cladogram used which is dependent on the characters chosen for the phylogenetic analysis. Though these results are very preliminary, it raises the interesting question of are progressive macroevolutionary patterns more common in colonial organisms. 